\title{
The role of cortisol and thyroid stimulating hormone in prognosis of acute anticholinesterase pesticides poisoned patients admitted to Tanta Poison Control Center
}

\author{
Wafaa M. Masoud, Mona Mohammed Heshmat, Nema A. Soliman, Heba K. Khalifa. ${ }^{1}$ \\ ${ }^{1}$ Department of Forensic Medicine and Clinical Toxicology, Faculty of Medicine Tanta University, Tanta, Egypt.
}

\begin{tabular}{ll}
\hline Abstract & Introduction: Anticholinesterase poisoning is a major global health problem with thousands of \\
deaths each year. Determination of prognosis is a major concern for clinical toxicologists. \\
Previous studies reported that cortisol and thyroid stimulating hormone (TSH) may have \\
prognostic role in patients poisoned by organophosphates. Aim of the work: Evaluation of \\
prognostic role of cortisol and TSH in acute anticholinesterase pesticides poisoned patients \\
admitted to Tanta Poison Control Center. Methodology: This prospective study included 29 \\
patients of moderate to severe acute anticholinesterase poisoning according to poisoning severity \\
score (PSS) between August 2019 and July 2020. TSH and serial cortisol were assessed by \\
ELISA techniques. Results: TSH has no prognostic role, but cortisol has predictive role in \\
mortality, need for ICU admission and intubation. Conclusion: Measuring cortisol at admission, \\
after six and twelve hours from admission could predict need for ICU admission, intubation and \\
mechanical ventilator, while measuring it at admission can be predictor for mortality. \\
Received in original form: 27 June 2021 Accepted in a final form: 5 October 2021 \\
Key words & Organophosphates, pesticides, TSH, cortisol, prognosis \\
\hline
\end{tabular}

\section{Introduction}

A nticholinesterase pesticides are common to be used in Egypt due to their wide availability and cheap prices. They are considered to be frequent reasons for admission in hospitals and intensive care units (ICU) in developing countries (Arafa et al., 2017 \& Elagamy and Gaber, 2019)

Organophosphorous and carbamates poisoning cause acetylcholinesterase (AChE) inhibition at cholinergic synapses at the central nervous system and autonomic nervous system, as well as at the neuromuscular junction (NMJ) resulting in excess sweating, salivation, bronchospasm, bronchorrhea, bradycardia, NMJ dysfunction and disturbed consciousness (Arafa et al., 2017).

During critical illness, profound changes may occur in the hypothalamic-pituitary-thyroid (HPT) and hypothalamic-pituitary-adrenal axes (HPA) with increase in cortisol level. At the level of the hypothalamus, thyrotropin-releasing hormone (TRH) is released and stimulates the thyrotropic cells in the pituitary to synthesize and secrete thyroid-stimulating hormone (TSH). TSH in turn drives the thyroid gland to synthesize and secrete thyroid hormones (Abo Elfottoh et al., 2020).

Specifically, Rao and Bhavana (2015) reported that human thyroid has cholinergic innervation.

Acetylcholine (Ach) as a neurotransmitter has been detected in the human body including the brain, vascular system, urogenital system, and endocrine system. Anticholinesterase can affect the endocrine system through hormone receptors, synthesis, and transcription factors. They exert their effects on pituitary hormones in the acute and chronic periods by decreasing cholinesterase levels (Şimşek et al., 2019).

Several animal studies revealed that cholinesterase inhibition causes endocrine disruption, affect adrenal gland steroidogenesis and Adrenocorticotropic hormone (ACTH) secretion so, affect cortisol level (Goad et al, 2004).

Determination of prognosis is a major concern for clinical toxicologists managing acutely poisoned patients. Prognostic factors facilitate appropriate disposition to limited ICU beds. (Alizadeh et al., 2014).

Therefore, the aim of the present study was to evaluate the prognostic role of cortisol and thyroid stimulating hormone (TSH) in acute anticholinesterase pesticides poisoned patients admitted to Tanta Poison Control Center.

\section{Patients and Methods}

Patients: This study was a prospective cohort study conducted on patients suffering from acute anticholinesterase pesticides poisoning who were admitted to the Poison Control Center of Tanta University Emergency Hospital, Tanta, Egypt, between August 2019 to end of July 2020. The study was approved by the Research Ethics Committee of the Faculty of Medicine, Tanta University. An informed written consent was obtained from each patient (or his attending relatives if unable to do so) after receiving 
detailed information about the study (Research ethics committee approval number: 33251/7/19) and data was collected through a standardized checklist. Privacy and confidentiality of patients and results of investigations were maintained by using coding number.

Inclusion criteria:

Patients of both sexes with moderate to severe symptoms of acute anticholinesterase poisoning according to poisoning severity score (PSS) (Persson et al., 1998) with diagnosis made on the basis of:

1. History taking either from patient himself or relatives.

2. Identification of the compound based on the container brought by patient or attendants if possible.

3. Manifestations of anticholinesterase poisoning as excess sweating, salivation, bronchospasm, bronchorrhea, bradycardia, hypotension, neuromuscular junction dysfunction (Tattersall, 2018).

4. Psudocholinesterase activity.

5. (Normal values at $37^{\circ} \mathrm{C}$ : in Children, men of all ages and women over 40 years: 5400- 13200 U/L, Women 18-39 years: 4300-11500 U/L) (Blawen et al., 1983).

Exclusion criteria:

1. Asymptomatic and mild cases.

2. Patients with history of co-ingestion.

3. Patients with history of thyroid disease.

4. Patients on steroid therapy

5. Pregnant and lactating women.

6. Patients with chronic and psychiatric diseases.

7. Conditions that affect pseudocholinesterase activity as malignancy, liver/kidney disease, pregnancy, burns, obesity, chronic alcoholism and medications as aspirin, metoclopramide, monoamine oxidase inhibitors and oral contraceptives (Lee et al., 2013).

Methodology: all cases were subjected to:

- History taking: Sociodemographic data including age and sex, toxicological history includes route of administration either inhalation, ingestion or dermal exposure, amount of pesticides taken (in case of ingestion), mode of poisoning either suicidal or accidental, time delay between pesticides exposure and hospital admission and any prehospital intervention.

- Clinical examination: Physical examination as vital signs (pulse, blood pressure, respiratory rate and temperature), level of consciousness assessed by Glasgow coma scale and systemic examination including neurological, respiratory, cardiovascular and abdominal examinations.

The normal values of vital signs are: Pulse ranges between 60 to 100 beat per minute, blood pressure ranges between $90 \backslash 60 \mathrm{mmhg}$ to $140 \backslash 90 \mathrm{mmhg}$, respiratory rate ranges between 12 to 20 breaths per minute (Hoffman et al., 2011) and temperature ranges between 36.5 to $37.5^{\circ} \mathrm{c}$ (Nield and Kamat, 2011).

- Laboratory investigations: includes arterial blood gases, serum sodium and potassium levels, random blood sugar, pseudocholinesterase activity, serum cortisol level assessed by ELISA technique (serial measurements on admission, after 6 hours and after 12 hours) and serum thyroid stimulating hormone
(TSH) level on admission assessed by ELISA technique.

- Electrocardiogram (ECG).

- Outcome measures (prognosis): Improved or died, need for ICU admission and assisted ventilation, ICU admission duration, total hospital admission duration and total needed amount of antidote therapy.

Criteria needed for ICU admission and assisted ventilation are: respiratory failure, ventricular arrhythmia and unstable hemodynamics (Sungur and Güven, 2001).

- Treatment: Started by providing an adequate airway, oxygenation, and ventilation and at stabilizing cardiorespiratory function by reversing excessive muscarinic effects. Once the patient become stable, the administration of an AChE-reactivating oxime drug, such as pralidoxime or obidoxime, can be considered along with the need for skin and/or gastric decontamination (Eddleston, 2019).

Statistical analysis

Statistical analysis was performed using Statistical Package for Social Sciences (IBM SPSS Statistics) for Windows, version 26 (IBM Corp., Armonk, N.Y., USA). For quantitative data, the Shapiro-Wilk test for normality was performed. For data that followed normal distribution, values were expressed as mean \pm standard deviation. For data that did not follow normal distribution, median, interquartile range (expressed as $1^{\text {st }}$ and $3^{\text {rd }}$ quartiles), and range (expressed as minimum to maximum) were calculated.

\section{Results}

In this study, there were twenty-nine acute anticholinesterase pesticides poisoned patients.

Table (1) shows the sociodemographic and toxicological data of our studied patients. Majority of cases $(69 \%)$ were below twenty years old (16-19 years) and $58.6 \%$ of them came from rural areas. The predominant sex was female $(62.1 \%)$. Concerning toxicological history, all cases ingested pesticides with suicidal intention and the mean time delay was 3.2 hours.

As regards clinical examination of our included patients presented in table (2), all of them had normal blood pressure. Sixty-five and half percent $(65.5 \%)$ of patients had normal heart rate and the rest of cases presented with tachycardia on admission. Normal respiratory rate and normal blood sugar were found in $72.4 \%$ and $86.2 \%$ of our patients respectively. As regards conscious level, $93.1 \%$ of patients were fully conscious. Two patients presented with disturbed conscious level (GCS 7\15). Chest findings showed that four patients had chest crepitation and two patients had wheezy chest. Cardiovascular and abdominal examinations were normal in all cases.

As regards investigations, normal $A B G$ and normal sodium level were found in majority of our patients with percentage of $69 \%$ and $93.1 \%$ respectively. Abnormalities in $\mathrm{ABG}$ results were detected in $31 \%$ of patients; in the form of respiratory alkalosis observed in $10.3 \%$ and respiratory acidosis in $6.9 \%$ of patients. Concerning potassium level, 
hypokalemia was observed in majority of studied patients $(58.6 \%)$. Mean pseudocholinesterase activity was $1100 \pm 800 \mathrm{U} / \mathrm{L}$.

Tables $(3,4)$ show the levels of measured hormones of studied patients; median serum TSH was $1.14 \mathrm{mIU} / \mathrm{L}$. Regarding cortisol, by comparing data in the three measurements, there was significant difference when comparing first and second measurements and when comparing first and third measurements ( $P$ value was $<0.001)$.

Normal ECG was found in $93.1 \%$, of patient and $6.9 \%$ of patients had sinus tachycardia.

As regards outcome, only two patients died (6.9\%) because of respiratory failure and four patients $(13.8 \%)$ needed ICU admission, intubation and mechanical ventilation due to bronchorea and respiratory muscles weakness that caused hypoxia. The mean ICU admission duration was 48 hours and the mean of total hospital admission duration was $39 \pm 29$ hours. Concerning PSS, only two cases (6.9\%) had severe toxicity. Rest of patients had moderate toxicity according to this scale.

Tables $(5,6)$ show the relationship between levels of TSH, cortisol hormones and mortality, there was no significant difference between survivors and non survivors as regards TSH level (P value was $>0.05$ ). As regards cortisol, there was significant difference between survivors and non survivors in the first (on admission) estimation only ( $P$ value was $<0.05$ ).

With concern to relationship between measured hormones and need for ICU admission and assisted ventilation, there was no significant difference in patients needed ICU admission and assisted ventilator and patient didn't need regarding TSH level ( $P$ value was $>0.05)$ as shown in table (7). Moreover, there was significant difference between them regarding the three serial cortisol estimations ( $P$ value were $<0.05$ ).

As regards relationship between hormones and pseudocholinesterase activity, there was only significant association with cortisol level after 6 hours $(P$ value $<0.05)$. While correlation between level of hormones and total antidote therapy given showed that there was only significant association between total number of atropine ampules and second measurement of serum cortisol level ( $P$ value was <0.05) as mentioned in table (9).

Table (10) and figure (1) show the results of ROC curve of serum cortisol level at admission as predictor for mortality in anticholinesterase pesticides poisoned patients. It had AUC of 1 which is graded as excellent one. The optimum cut-off value was identified at $>$ $80.9 \mu \mathrm{g} / \mathrm{dl}$. So at this point, cortisol level had a sensitivity and specificity of $100 \%$.

As regards need for ICU admission, intubation and mechanical ventilation, the second and third cortisol measurements had AUC of 1 which is graded excellent. The optimum cutoff value for both measurements were identified at $>62.7$ and $>30.2 \mu \mathrm{g} / \mathrm{dl}$ respectively, so at this level they had $100 \%$ specificity and sensitivity as shown in figures (2-4) and table (11).

Table (1): Distribution of sociodemographic and toxicological data of 29 cases of anticholinesterase pesticides poisoning included in the study:

\begin{tabular}{|c|c|c|c|c|c|c|c|c|c|c|c|c|c|c|}
\hline & \multicolumn{3}{|c|}{$\underset{<}{\infty}$} & \multicolumn{2}{|c|}{$\stackrel{\star 凶}{\varpi}$} & \multicolumn{2}{|c|}{ 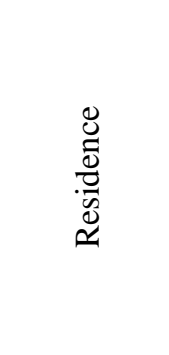 } & \multicolumn{2}{|c|}{ 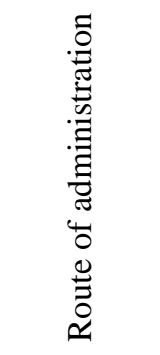 } & \multicolumn{2}{|c|}{ 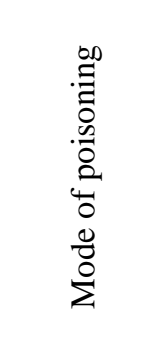 } & \multicolumn{2}{|c|}{ 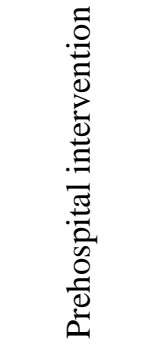 } & \multirow{2}{*}{ 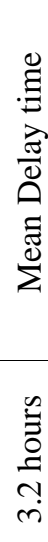 } \\
\hline & $\frac{a}{b}$ & તે & $\hat{n}$ & $\frac{0}{\tilde{J}}$ & 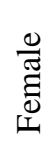 & 䒕 & 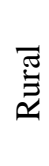 & 퓸 & 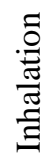 & 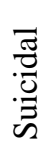 & 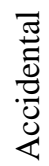 & $\stackrel{\infty}{2}$ & $\stackrel{\circ}{Z}$ & \\
\hline $\begin{array}{c}\text { Number of } \\
\text { cases / } \\
\text { percentages }\end{array}$ & $\begin{array}{l}\text { å } \\
\text { aे } \\
\text { वे}\end{array}$ & $\frac{a}{a}$ & 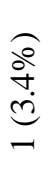 & $\begin{array}{l}\widehat{\rho} \\
a \\
\hat{n} \\
= \\
=\end{array}$ & 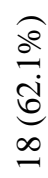 & $\begin{array}{l}\overparen{d} \\
\underset{J}{d} \\
\stackrel{d}{d}\end{array}$ & $\begin{array}{l}\widehat{o} \\
\delta \\
\infty \\
0 \\
0 \\
=\end{array}$ & $\begin{array}{l}\text { o } \\
8 \\
\stackrel{8}{8} \\
\text { ते }\end{array}$ & $\overbrace{0}^{2}$ & $\begin{array}{l}\text { o̊ } \\
8 \\
8 \\
\text { ㄱ. }\end{array}$ & $\frac{0}{e}$ & 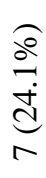 & 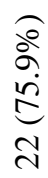 & \\
\hline
\end{tabular}


Table (2): Distribution of vital signs and level of consciousness among 29 cases of anticholinesterase pesticides:

\begin{tabular}{|c|c|c|c|c|c|c|c|c|c|c|c|}
\hline & \multicolumn{2}{|c|}{ Pulse } & \multicolumn{2}{|c|}{ Blood pressure } & \multicolumn{3}{|c|}{ Respiratory Rate } & \multicolumn{2}{|c|}{ Random Blood Sugar } & \multicolumn{2}{|c|}{$\begin{array}{c}\text { Level of } \\
\text { Consciousness }\end{array}$} \\
\hline & $\begin{array}{l}\overline{\tilde{F}} \\
\overline{\tilde{0}} \\
\text { Z }\end{array}$ & 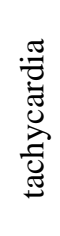 & $\begin{array}{l}\overline{\widetilde{\Xi}} \\
\text { है } \\
\text { Z }\end{array}$ & 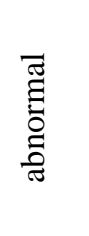 & $\begin{array}{l}\bar{\Xi} \\
\tilde{E}\end{array}$ & 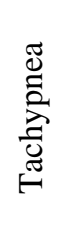 & 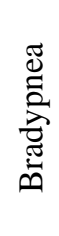 & $\begin{array}{l}\bar{\Xi} \\
\bar{E} \\
z\end{array}$ & 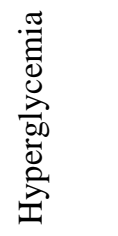 & 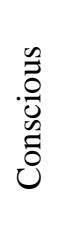 & ப் \\
\hline $\begin{array}{c}\text { Number of cases } \\
\text { / percentages }\end{array}$ & $\begin{array}{l}\widehat{o} \\
n \\
i \\
2 \\
a\end{array}$ & $\begin{array}{l}\widehat{Q} \\
\stackrel{n}{2} \\
\stackrel{0}{0} \\
0\end{array}$ & 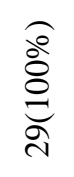 & $e_{0}^{8}$ & $\begin{array}{l}\widehat{d} \\
\stackrel{d}{+} \\
\stackrel{a}{\Xi} \\
\vec{d}\end{array}$ & $\begin{array}{l}\widehat{o} \\
\stackrel{0}{0} \\
\text { d } \\
0\end{array}$ & $\begin{array}{l}\text { बे } \\
\text { aे } \\
\text { v }\end{array}$ & 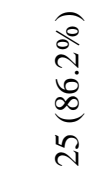 & $\begin{array}{l}\underset{\theta}{0} \\
\infty \\
\stackrel{+}{\Xi}\end{array}$ & $\begin{array}{l}\hat{\sigma} \\
\frac{\partial}{a} \\
\stackrel{\sim}{d}\end{array}$ & $\begin{array}{l}\text { ô } \\
\text { oे } \\
\text { va }\end{array}$ \\
\hline
\end{tabular}

Table (3): Thyroid stimulating hormone level (mIU/L) of 29 cases of anticholinesterase pesticides poisoning:

\begin{tabular}{|c|c|c|c|}
\hline \multicolumn{3}{|c|}{ Thyroid stimulating hormone (mIU/L) } \\
\hline Range & $1^{\text {st }}$ quartile & Median & $3^{\text {rd }}$ quartile \\
\hline $0.36-3.12$ & 0.96 & 1.14 & 1.51 \\
\hline
\end{tabular}

Table (4): Friedman test: statistical analysis to compare serial cortisol levels $(\mu \mathrm{g} / \mathrm{dl})$ for 29 poisoned cases by anticholinesterase pesticides:

\begin{tabular}{|c|c|c|c|c|c|}
\hline Cortisol $(\mu \mathrm{g} / \mathrm{dl})$ & At Admission & After 6 Hours & After 12 Hours & $X^{2}$ & P \\
\hline Range & $15.1-82.2$ & $9.17-64.50$ & $4.24-84.00$ & \multirow{2}{*}{15.600} & \multirow{2}{*}{$<0.001 *$} \\
\hline Median & 47.3 & 28.20 & 23.50 & & \\
\hline Post Hoc Dun Bonferrani & P1: $0.001 *$ & P2: 0.279 & P3: $0.001 *$ & & \\
\hline
\end{tabular}

$X^{2}:$ Friedman test, *: statistically significant result at $P$ value $<0.05, P 1:$ comparing level at admission with level at 6 hours, P2: comparing level at 6 hours with the level at 12 hours, P3: comparing level at admission with the level at 12 hours

Table (5): Mann whitey $U$ test: statistical analysis to compare between survivors and non-survivors among 29 cases of anticholinesterase pesticides poisoning included in this study regarding TSH level (mIU/L):

\begin{tabular}{|c|c|c|c|c|}
\hline \multirow{2}{*}{ TSH (mIU/L) } & \multicolumn{2}{|c|}{ Outcome } & \multirow{2}{*}{$\mathrm{Z}$} & $\mathrm{P}$ \\
\cline { 2 - 5 } & $\begin{array}{c}\text { Survivors } \\
\mathrm{N}=27\end{array}$ & $\begin{array}{c}\text { Non-survivors } \\
\mathrm{N}=2\end{array}$ & -1.126 & 0.260 \\
\hline 1st quartile & 0.96 & 1.51 & & \\
\hline Median & 1.14 & 1.51 & 1.51 & \\
\hline 3rd quartile & 1.67 & & & \\
\hline
\end{tabular}

TSH: thyroid stimulating hormone, Z: Mann whitey U test, *: statistically significant result (P value <0.05)

Table (6): Mann whitey $U$ test: statistical analysis to compare between survivors and non-survivors among 29 cases of anticholinesterase pesticides poisoning included in this study regarding serial cortisol measurements ( $\mu \mathrm{g} / \mathrm{dl})$ :

\begin{tabular}{|c|c|c|c|c|}
\hline \multirow[b]{2}{*}{ Cortisol $(\mu \mathrm{g} / \mathrm{dl})$} & \multicolumn{2}{|c|}{ Outcome } & \multirow[b]{2}{*}{$\mathrm{Z}$} & \multirow[b]{2}{*}{$\mathrm{P}$} \\
\hline & $\begin{array}{c}\text { Survivors } \\
\mathrm{N}=27\end{array}$ & $\begin{array}{l}\text { Non-survivors } \\
\quad \mathrm{N}=2\end{array}$ & & \\
\hline \multicolumn{5}{|l|}{ Cortisol at admission } \\
\hline 1st quartile & 32.9 & 82.2 & & \\
\hline Median & 47.3 & 82.2 & 2.328 & 0.020* \\
\hline 3rd quartile & 54.1 & 82.2 & & \\
\hline \multicolumn{3}{|l|}{ Cortisol after 6 hours } & \multirow{8}{*}{\multicolumn{2}{|c|}{$\begin{array}{c}\text { Not } \\
\text { Applicable }\end{array}$}} \\
\hline 1st quartile & 19.95 & - & & \\
\hline Median & 28.20 & - & & \\
\hline 3rd quartile & 39.95 & - & & \\
\hline \multicolumn{3}{|l|}{ Cortisol after 12 hours } & & \\
\hline 1st quartile & 18.20 & - & & \\
\hline Median & 23.50 & - & & \\
\hline 3rd quartile & 29.40 & - & & \\
\hline
\end{tabular}

Z: Mann whitey U test, *: statistically significant result ( $P$ value $<0.05)$ 
Table (7): Mann whitey $U$ test: statistical analysis for comparison between 29 cases of anticholinesterase pesticides poisoning who needed intensive care unit admission, intubation and mechanical ventilator and who didn`t need regarding TSH level $(\mathrm{mIU} / \mathrm{L})$ :

\begin{tabular}{|c|c|c|c|c|}
\hline \multirow{2}{*}{ TSH (mIU/L) } & \multicolumn{2}{|c|}{ ICU admission } & \multirow{2}{*}{ Z } & P \\
\cline { 2 - 5 } & $\begin{array}{c}\text { Need } \\
\mathrm{N}=4\end{array}$ & $\begin{array}{c}\text { Not need } \\
\mathrm{N}=25\end{array}$ & & \\
\hline 1st quartile & 1.14 & 0.96 & -0.763 & 0.445 \\
\hline Median & 1.33 & 1.14 & & \\
\hline 3rd quartile & 1.51 & 1.67 & & \\
\hline
\end{tabular}

ICU: intensive care unit, Z: Mann whitey U test

Table (8): Mann whitey $U$ test: statistical analysis for comparison between 29 cases of anticholinesterase pesticides poisoning who needed intensive care unit admission, intubation and mechanical ventilator and who didn't need regarding serial cortisol measurements $(\mu \mathrm{g} / \mathrm{dl})$ :

\begin{tabular}{|c|c|c|c|c|}
\hline \multirow[b]{2}{*}{ Cortisol $(\mu \mathrm{g} / \mathrm{dl})$} & \multicolumn{2}{|c|}{ ICU admission } & \multirow[b]{2}{*}{$\mathrm{Z}$} & \multirow[b]{2}{*}{$\mathrm{P}$} \\
\hline & $\begin{array}{l}\text { Need } \\
N=4\end{array}$ & $\begin{array}{c}\text { Not need } \\
\mathrm{N}=25\end{array}$ & & \\
\hline \multicolumn{5}{|l|}{ Cortisol at admission } \\
\hline 1st quartile & 54.1 & 32.9 & & \\
\hline Median & 68.2 & 45.0 & -2.407 & $0.016^{*}$ \\
\hline 3rd quartile & 82.2 & 53.0 & & \\
\hline \multicolumn{5}{|l|}{ Cortisol after 6 hours } \\
\hline 1st quartile & 64.50 & 17.40 & & \\
\hline Median & 64.50 & 28.10 & -2.304 & $0.021 *$ \\
\hline 3rd quartile & 64.50 & 31.20 & & \\
\hline \multicolumn{5}{|l|}{ Cortisol after 12 hours } \\
\hline 1st quartile & 84.00 & 18.20 & & \\
\hline Median & 84.00 & 21.90 & -2.276 & $0.023 *$ \\
\hline 3rd quartile & 84.00 & 27.40 & & \\
\hline
\end{tabular}

Z: Mann whitey U test, *: statistically significant result (P value <0.05), ICU: intensive care unit, Z: Mann whitey U test

Table (9): Pearson Correlation between total atropine and oximes ampoles given, cholinesterase activity (U/L) and

TSH (mIU/L) and serial cortisol $(\mu \mathrm{g} / \mathrm{dl})$ levels in 29 anticholinesterase poisoned patients:

\begin{tabular}{|c|c|c|c|}
\hline \multicolumn{2}{|c|}{ Total Atropine } & Total Oximes & Cholinesterase Activity (U/L) \\
\hline TSH $(\mathrm{mIU} / \mathrm{L})$ & \multicolumn{1}{|c|}{0.268} \\
\hline $\mathrm{R}$ & 0.130 & -0.084 & 0.160 \\
\hline $\mathrm{P}$ & 0.500 & 0.665 & 0.012 \\
\hline Cortisol at Admission $(\mu \mathrm{g} / \mathrm{dl})$ & 0.002 & 0.317 & 0.950 \\
\hline $\mathrm{R}$ & 0.994 & 0.094 & -0.439 \\
\hline $\mathrm{P}$ & 0.423 & 0.342 & $0.032^{*}$ \\
\hline Cortisol after 6 Hours $(\mu \mathrm{g} / \mathrm{dl})$ & $0.039^{*}$ & 0.102 & -0.186 \\
\hline $\mathrm{P}$ & -0.367 & 0.190 & 0.432 \\
\hline Cortisol after 12 Hours $(\mu \mathrm{g} / \mathrm{dl})$ & 0.178 & 0.422 & \\
\hline $\mathrm{R}$ &
\end{tabular}

r: Pearson Correlation, * Statistically significant $(<0.05)$

Table (10): ROC curve analysis: Serum cortisol hormone ( $\mu \mathrm{g} / \mathrm{dl})$ at admission as predictor of mortality in anticholinesterase pesticides poisoned patients ( 29 cases):

\begin{tabular}{|c|c|c|c|c|c|}
\hline Parameters & $\begin{array}{c}\text { AUC } \\
(95 \% \mathrm{CI})\end{array}$ & $\mathrm{p}$ - value & Cut-off value & Sensitivity $(\%)$ & Specificity $(\%)$ \\
\hline Cortisol at admission $(\mu \mathrm{g} / \mathrm{dl})$ & $1.000(0.884-1.000)$ & $<0.001 *$ & $>80.9$ & 100.0 & 100.0 \\
\hline
\end{tabular}

AUC: area under the curve; CI: confidence interval 
Table (11): ROC curve analysis: Comparison between cortisol $(\mu \mathrm{g} / \mathrm{dl})$ at admission, 6 hours and twelve hours as predictors of ICU admission, intubation and mechanical ventilator for anticholinesterase pesticides poisoned cases (29 cases)

\begin{tabular}{|c|c|c|c|c|c|}
\hline Parameters & $\begin{array}{c}\text { AUC } \\
(95 \% \mathrm{CI})\end{array}$ & $\mathrm{p}$ - value & Cut off value & Sensitivity (\%) & Specificity (\%) \\
\hline $\begin{array}{c}\text { Cortisol at admission } \\
(\mu \mathrm{g} / \mathrm{dl})\end{array}$ & $\begin{array}{c}0.885 \\
(0.715-0.972)\end{array}$ & $<0.001 *$ & $>53$ & 100.0 & 76.9 \\
\hline $\begin{array}{l}\text { Cortisol after } 6 \text { hours } \\
(\mu \mathrm{g} / \mathrm{dl})\end{array}$ & $\begin{array}{c}1.000 \\
(0.858-1.000)\end{array}$ & $<0.001 *$ & $>62.7$ & 100.0 & 100.0 \\
\hline $\begin{array}{l}\text { Cortisol after } 12 \\
\text { hours }(\mu \mathrm{g} / \mathrm{dl})\end{array}$ & $\begin{array}{c}1.000 \\
(0.832-1.000)\end{array}$ & $<0.001 *$ & $>30.2$ & 100.0 & 100.0 \\
\hline $\begin{array}{l}\text { p-value from Pairwise } \\
\text { comparisons }\end{array}$ & & $\begin{array}{r}\text { Admissio } \\
\text { Admissior } \\
\text { 6-hr c }\end{array}$ & $\begin{array}{l}\text { tisol vs } 6 \text {-hr ce } \\
\text { isol vs } 12-\mathrm{hr} \\
1 \mathrm{vs} 12-\mathrm{hr} \text { cor }\end{array}$ & $\begin{array}{l}\mathrm{ll}=0.004^{*} \\
\mathrm{ol}=0.004^{*} \\
=1.000\end{array}$ & \\
\hline
\end{tabular}

AUC: area under the curve; CI: confidence interval; * significant at $p<0.05$.

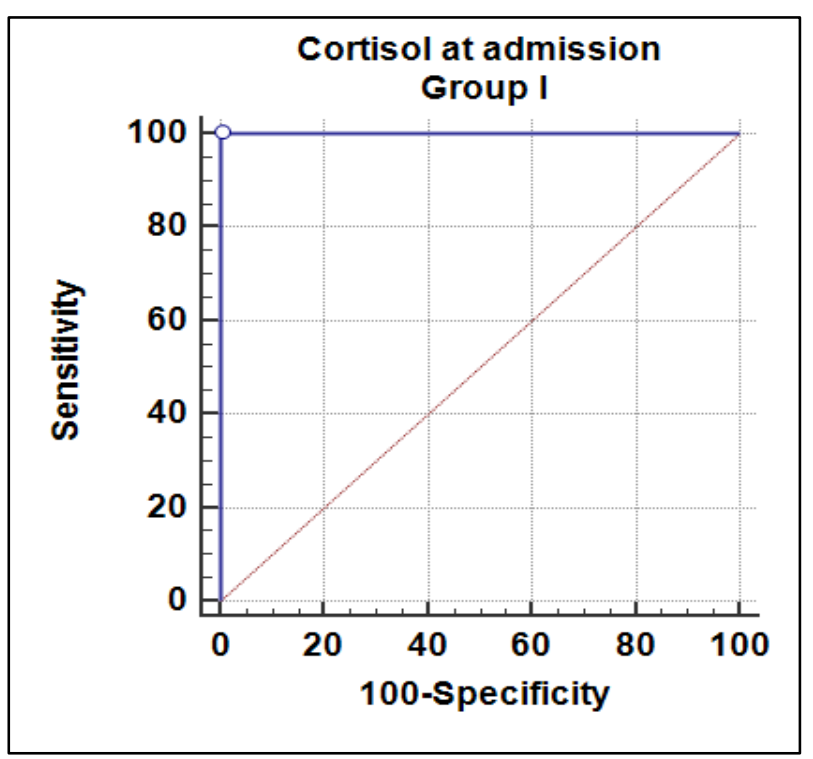

Figure (1): Relationship between outcome and cortisol at admission for 29 cases of anticholinesterase pesticides poisoned patients

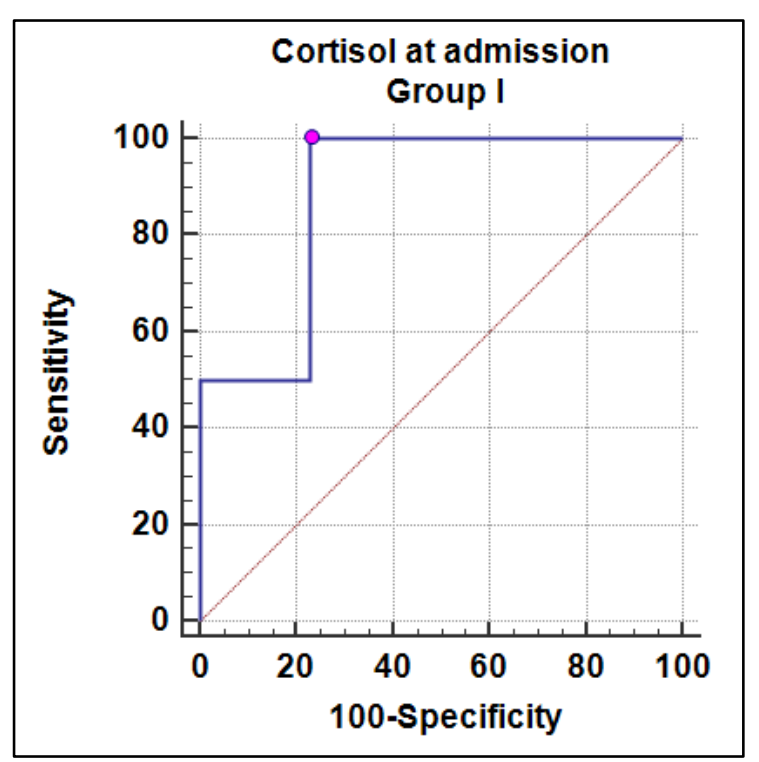

Figure (2): Relationship between cortisol at admission and need for ICU admission for anticholinesterase pesticides poisoned cases (29 cases) 


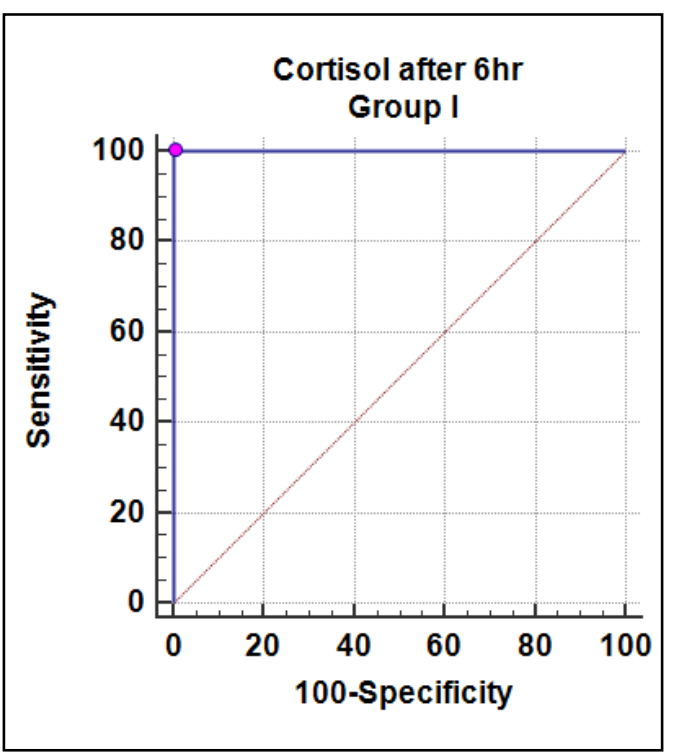

Figure (3): Relationship between cortisol after six hours from admission and need for ICU admission, intubation and mechanical ventilator for anticholinesterase pesticides poisoned cases ( 29 cases)

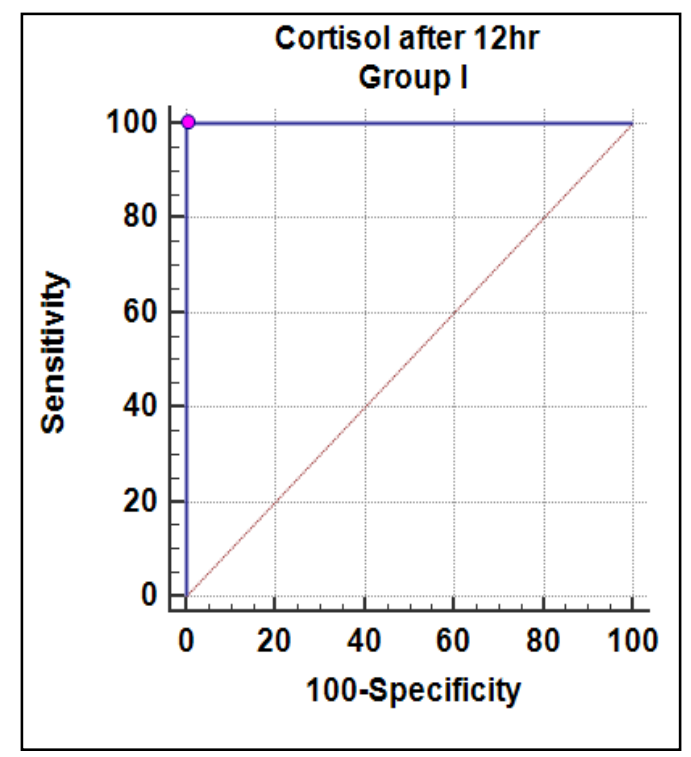

Figure (4): Relationship between cortisol after twelve hours from admission and need for ICU admission, intubation and mechanical ventilator for anticholinesterase pesticides poisoned cases ( 29 cases)

\section{Discussion}

In Egypt, anticholinesterase poisoning constitutes the majority of suicidal cases by pesticides due to their easy availability and low price (Gunnell et al., 2007). Concerning Tanta Poison Control Center, El-Maddah, (2012) and Abo El-Noor, (2013) reported that anticholinesterase pesticides poisoned patients represented $30.4 \%$ and $20 \%$ of acutely poisoned patients respectively. Furthermore, El-Mansy et al, (2014) revealed that phosphide poisoning represent the most common cause of death followed by anticholinesterase poisoning in the period between 2009 and 2013.

Determination of prognosis may allow more intensive monitoring and favorable outcome in pesticides poisoned patients (El-Sarnagawy, 2017).
Previous studies reported that cortisol and TSH may have prognostic role in organophosphates poisoned patients (Shadnia et al., 2018).

Changes in thyroid profile may occur in critical illnesses through different mechanisms such as the inhibition of thyroxine uptake by the hepatocytes and limiting its conversion to triiodothyronine in the periphery, also circulating cytokines can influence hormones levels. These alterations in thyroid hormones levels are called sick euthyroid syndrome. This syndrome may result from severe illness, physical trauma, physiologic stress and large number of drugs and chemical compounds as some pesticides (Tomescu et al., 2018). The normal TSH in healthy adult ranged from 0.5 to $5 \mathrm{mIU} / \mathrm{L}$ (Vadiveloo et al., 2013). 
Upon exposure to stressful conditions or in cases of critical illness, activation of hypothalamic-pituitaryadrenal axis (HPA) occurs with subsequent increase in cortisol level (Herman et al., 2016). However in some patients, their cortisol production is not sufficiently increased in response to stress conditions which is known as critical illness-related corticosteroid insufficiency (CIRCI) (Pisano et al., 2017).

According to Kronenberg, (2008), the normal cortisol levels during stress should be more than 34 $\mu \mathrm{g} / \mathrm{dl}$.

In addition, Anticholinesterase pesticides can affect the endocrine system through hormone receptors, hormone synthesis, and transcription factors (Şimşek et al., 2019). Based on that, our study was made to evaluate the prognostic role of and cortisol and thyroid stimulating hormones in acute pesticides poisoned patients.

The present study included 29 patients with acute anticholinesterase pesticides poisoning. All included patients were at middle age ranged between 16 and 37 years old as this age group is the most physically active and more liable to stress and depression during their lives (Farahat et al., 2016). Also, we found that all included patients ingested pesticides with suicidal intension according to their allegation. This could be attributed to many factors such as poverty, financial problems, economic instability, unemployment, marital conflicts, failure of love and breakup in the family support system (Kumar et al., 2010). Besides, many individuals do not seek help for their suicidal thoughts because of the stigma associated with mental illness ( $\mathrm{Li}$ et al., 2015). These results are similar to El-Gendy et al., (2017) who reported that majority of their patients were at middle age and ingested pesticides with suicidal intention.

As regarding sex, there was female predominance in this study representing $62 \%$ of patients. This may be due to sociocultural factors as lower education and lower economic income than males (Arafat, 2019).

These results come in line with Sarhan, (2015) and Ohbe et al., (2018) results. Moreover, this is contradictory to El-Sheikh et al., (2017) who found $83 \%$ of patients were males and El-Gendy et al., (2017) who reported that $57.5 \%$ were males. They explain that as males work in agriculture more than females.

Regarding residence, $58.6 \%$ of patients were from rural areas. This is similar to Elgohary et al., (2013) and El-Gendy et al., (2017) who found $82 \%$ and $60 \%$ of their patients from rural areas explaining that by wide exposure to these compounds in the Delta agriculture region.

Regarding time delay between pesticides exposure and hospital admission, the median time delay in this study was 3.2 hours. This is due to presence of Tanta Poison Control Center near to rural areas in Gharbia Governorate. This come in line with Oreby and Elmadah (2017) result who made their study in Tanta also. This is different to Phanindra et al., (2020) in India who reported that mean time delay in their patients was 6 hours.

However, according to Liu et al., (2015) time between anticholinesterase exposure and seeking medical advice could be attributed to nature of poisoning, awareness of family by toxicity, availability of transportation and distance of hospitals from poisoning place.

In this study, all patients had normal blood pressure, $34.5 \%$ had tachycardia. This is explained by Rabha et al., (2017) as preganglionic nicotinic receptor stimulation was followed by release of adrenaline and noradrenaline from adrenal gland leading to predominance of adrenergic activity on the heart. Moussa et al., (2018) found that $9.5 \%$ of patients presented with tachycardia and $1.5 \%$ of patients presented with bradycardia. Concerning the blood pressure, they found $5 \%$ of their patients had hypotension, $2 \%$ of patients had hypertension. Also, Farooqui et al., (2020) and Luo et al., (2020) reported normal blood pressure in majority of their cases. Banerjee et al., (2012) reported occurrence of hypertension in $9 \%$, bradycardia in $38 \%$ and tachycardia in $8 \%$. These differences between the studies may be caused by differences in delay time before hospital admission. Gündüz et al., (2015) noted that after exposure to anticholinesterase, initial tachycardia occurs due to sympathetic stimulation followed by bradycardia due to parasympathetic stimulation.

Regarding respiratory rate, abnormalities were recorded in $27.6 \%$ of patients $(20.7 \%$ had tachypnea, $6.9 \%$ with bradypnea). Other studies as Banerjee et al., (2012) and Moussa et al., (2018) reported that tachypnea represented $6 \%, 35 \%$ of cases respectively. Tachypnea may be a sign of respiratory failure (Sungur and Güven, 2001). Carey et al., (2013) reported that accumulated Ach at carotid body chemoreceptors increases respiratory activity.

Chest examination revealed $13.8 \%$ of cases had chest crepitations, $6.9 \%$ had wheezy chest. This is partially similar to Karki et al., (2004) who found chest abnormalities in $21.6 \%$ of their patients. Abedin et al., (2012) reported that $70 \%$ of their patients had chest manifestations as wheeze and crepitations. In addition, Elagamy and Gabr, (2019) revealed presence of bronchorhea in 58\% in their study group.

Respiratory manifestations are main signs of anticholinesterase pesticides poisoning which are a part of acute cholinergic crisis occurred by acetylcholine accumulation (Hulse et al., 2014).

Regarding serum potassium level, there was hypokalemia in $58.6 \%$ of patients, which explained by vomiting and diarrhea which are very common muscarinic manifestations (Roberts and Brett, 2014). This is partially similar to Abd Alkareem and Khater, (2019) who reported hypokalemia in $41.6 \%$ of their patients. On the other hand, (Someswar et al., 2015) found hypokalemia in $24 \%$ of their patients. They explained the bad prognosis associated with hypokalemia could be explained by muscle weakness, cardiac dysrhythemia and renal failure.

Concerning ECG, it was normal in $93.1 \%$ of cases in this study, only $6.9 \%$ had sinus tachycardia. Uddin et al., (2020) reported normal ECG findings in $64 \%$ of their patients and the commonest change was sinus tachycardia in $14 \%$ of cases.

In this study, only $6.9 \%$ of cases died and $13.8 \%$ were intubated and admitted to ICU. This percentage of 
mortality may be explained by early administration to hospital with early intervention with antidotes. In addition, because $93.1 \%$ were moderate cases as regards poison severity score. This mortality percentage was lower than El-Gendy et al., (2017), Amin et al., (2018) and Oreby and Elmadah, (2017), also the mean ICU stay duration was 48 hours and total hospital stay time was 39 hours in this study. Same results obtained by Brvar et al., (2018). However, Samprathi et al., (2020) mean hospital stay in their patients was 9.4 days and ICU stay was 5.9 days.

During critical illness, profound changes may occur in the hypothalamic-pituitary-thyroid (HPT) axis. The most consistent change is a decrease in serum triiodothyronine (T3) level, but in severe illness, serum thyroxine (T4) and TSH may also decrease (Fliers et al., 2001).

Anticholinesterase compounds affect thyroid hormones through different mechanisms including interfering with their biosynthesis, interrupting the transport of thyroid hormones in the blood circulation, their binding to receptors and disrupting their metabolism in the liver (Yang et al., 2020).

Concerning TSH level, majority of patients had normal level; only one patient had decreased its level. This come in line with Güven et al., (1999), Dutta et al., (2015) and Gundogan et al., (2018). This may be explained by euthyroid sick syndrome which is characterized by normal TSH, T4 levels and decreased T3 level which normalize after treatment (Tomescu et al., 2018).

Furthermore, Hundekari et al., (2012) made a study on 150 organophosphate poisoned patients, they reported that there was a slight and nonsignificant decrease in serum T3 and serum T4 levels in OP poisoned cases without any significant change in serum TSH levels as compared to control and their values didn`t change after treatment. Moreover, Rao and Bhavana (2015) discussed two case reports in which there was decreased TSH measurement and hyperthyroidism at stage of acute poisoning and normalized at time of discharge.

Dutta et al., (2015) revealed that alteration in TSH level during critical illness depend on severity and duration of illness, age of the patient and cytokines level. This didn't occur in our study may be due to majority of our patients were at middle age and were moderate cases as regard PSS. Also, we selected only acute poisoning with anticholinesterase pesticides.

Another study as Satar et al., (2008) reported histological structural changes in thyroid gland after poisoning with methamidophos (one of OP compounds) suggesting that certain OP compound may have toxic effect on thyroid gland. Furthermore, Huang et al., (2017) concluded increased risk of hypothyroidism after anticholinesterase poisoning especially in age group 40-64 years old. This difference between our study and their study because our patients were at middle age.

Regarding prediction of mortality by TSH level, this study showed no significant difference between survivors and non-survivors. This comes in line with Najari et al., (2018) results. Also, regarding secondary outcome as need for intubation or ICU admission, this study reported that TSH level can`t predict need for intubation or ICU admission.

As regards serum cortisol level, this study revealed that median cortisol level at admission was $47.3 \mu \mathrm{g} / \mathrm{dl}$. This is partially similar to Satar, (2004) who reported median cortisol level at admission was $41.8 \mu \mathrm{g} / \mathrm{dl}$. Other studies as Güven et al., (1999) and Dutta et al., (2015) reported that the mean cortisol level at admission was $25.5 \pm 14$ and $34.3 \mu \mathrm{g} / \mathrm{dl}$ respectively. The initial rise in cortisol level is explained by stress or the effect of acetylcholine on corticotropin releasing hormone and ACTH (Kale, 2014).

According to Kronenberg, (2008), if blood cortisol level less than $15 \mu \mathrm{g} / \mathrm{dl}$ is graded as adrenal insufficiency, if between 15 and $33 \mu \mathrm{g} / \mathrm{dl}$ is graded as CIRCI and if more than $34 \mu \mathrm{g} / \mathrm{dl}$ is considered adequate adrenal response to stress.

Previous studies reported that cortisol level increased during stressful conditions and that increase is related to severity of illness, loss of circadian level, increased its production and decrease in its half-life and they also reported that loss of AChE activity is similar to a generalized stress reaction, in which the plasma cortisol concentration should elevate (Satar, 2004).

Furthermore, Martin-Reina et al., (2017) reported that acute anticholinesterase poisoning might cause significant elevation of prolactin, adrenocorticotrophic and cortisol. Güven et al., (1999) noted in their study that numerous animal studies were done and reported increased cortisol levels in acute anticholinesterase poisoning and shown that the degree of elevation is related to the severity of illness.

In this study, we measure cortisol level at three stations; first at admission, second estimation after six hours and the last measurement after twelve hours of admission. During this period, first aid was made and patients received their antidote therapy. According to Kronenberg, (2008), majority of cases (74.1\%) showed adequate adrenal response to stress at admission, moreover after six and twelve hours incidence of critical illness related corticosteroid insufficiency increased represented $48.1 \%$ and $59.3 \%$ respectively.

By comparing cortisol level at admission with mortality, we had significant difference between survivors and non-survivors. Also, this study reported that there was significant difference between the three cortisol maesurements and need for ICU admission and intubation.

\section{Conclusion}

This study concluded that measurement of cortisol at admission is a predictor for mortality in anticholinesterase pesticides poisoned patients. In addition, measuring cortisol at admission, after six and twelve hours from admission can predict need for ICU admission and intubation and mechanical ventilator but TSH have no prognostic role in organophosphates poisoned patients. 


\section{References}

Abd Alkareem M and Khater A (2019): Evaluation of Copeptin level and Peradeniya Score as Predictors of Severity and Outcome in Acute Organophosphorus Pesticides Poisoned Patients Admitted to the Poison Control Center Ain Shams University Hospitals (A Prospective Study). Ain Shams Journal of Forensic Medicine and Clinical Toxicology, 33(2), 104-112.

Abedin M J, Sayeed A A, Basher A, Maude R J, Hoque G and Faiz M A (2012): Open-label randomized clinical trial of atropine bolus injection versus incremental boluses plus infusion for organophosphate poisoning in Bangladesh. Journal of Medical Toxicology, 8(2), 108-117.

Abo Elfottoh M N, Kamal H M, Gab-allah A M and Ali E E S (2020): Clinical Evaluation of Hypothalamo-Pituitary-Thyroid Axis Hormones in Critically Ill Children: Cross Sectional Study at Zagazig University Children Hospital. The Egyptian Journal of Hospital Medicine, 81(2), 1525-1530.

Abo El-Noor M (2013): Pattern of Acute Poisoning among Patients Admitted to Tanta Poison Control Center during 2012. Ain Shams Journal of Forensic Medicine and Clinical Toxicology, 20(1), 158-169.

Alizadeh A M, Hassanian-Moghaddam H, Shadnia S, Zamani N and Mehrpour O (2014): Simplified acute physiology score II/acute physiology and chronic health evaluation II and prediction of the mortality and later development of complications in poisoned patients admitted to intensive care unit. Basicand Clinical Pharmacologyand Toxicology, 115 (3), 297-300.

Amin D M, Abaza M T, El Azawy D S and Ahmed A I (2018): Morbidity and Mortality Indicators in Acute Organophosphate Poisoning in Zagazig University Hospital, Egypt: Retrospective Study. Occupational Diseases and Environmental Medicine, 6(4), 130-140.

Arafa M H, Shalaby N M and Atteia H H (2017): Lactate and creatine phosphokinase as potential independent predictors of organophosphrus poisoning severity in Zagazig University Hospital Patients, Egypt. Journal of Toxicology and Environmental Health Sciences, 9(8), 73-82.

Banerjee I, Tripathi S K and Roy A S (2012): Clinicoepidemiological characteristics of patients presenting with organophosphorus poisoning. North American journal of medical sciences, 4(3), 147.

Blawen D, Poppe DH and Trischler WA (1983): Serum acetylcholinesterase assay. Journal of Clinical Chemistry and Clinical Biochemistry, 21(6): 381-386.

Brvar M, Chan M Y, Dawson A H, Ribchester R R and Eddleston M (2018): Magnesium sulfate and calcium channel blocking drugs as antidotes for acute organophosphorus insecticide poisoning-a systematic review and meta-analysis. Clinical Toxicology, 56(8), 725-736
Carey J L, Dunn C and Gaspari R J (2013): Central respiratory failure during acute organophosphate poisoning. Respiratory physiology and neurobiology, 189(2), 403-410.

Dutta P, Kamath S S, Bhalla A, Shah V N, Srinivasan A, Gupta P and Singh S (2015): Effects of acute organophosphate poisoning on pituitary target gland hormones at admission, discharge and three months after poisoning: A hospital based pilot study. Indian journal of endocrinology and metabolism, 19(1), 116.

Eddleston M (2019): Novel clinical toxicology and pharmacology of organophosphorus insecticide self-poisoning. Annual review of pharmacology and toxicology, 59, 341-360.

Eddleston M (2020): Poisoning by pesticides. Medicine, 48(3), 214-217, DOI: https://doi.org/ 10.1016/ j.mpmed.2019.12.019

Elagamy S E and Gabr H M (2019): Predictors of the need for Intensive Care Unit admission in acute organophosphorus poisoning: One year prospective study. The Egyptian Journal of Forensic Sciences and Applied Toxicology, 19(4), 1-9.

El-Gendy S A, El-Mehallawi I H, Elwy A M and ElGhrabawy I E S (2017): A randomized clinical trial to evaluate the efficacy of single versus multiple gastric lavage in the management of patients with acute organophosphorus poisoning. Tanta Medical Journal, 45(4), 181.

Elgohary M, El Ashmawy N, El Kelany R, Abo Elfadl A, and El-Sarnagawy G (2013): Comparative study of paraoxonase and cholinestrase enzymes activities in diagnosis of organophosphorus insecticide intoxication. Ain Shams Journal of Forensic Medicine and Clinical Toxicology, 21(2), 1-11.

El-Maddah E I (2012): Pattern of Acute Poisoning in Adult Patients Admitted to Tanta Poison CenterEgypt. The Egyptian Journal of Forensic Sciences and Applied Toxicology, 220(1245), 1-15.

EL-Mansy A M, Ahmed Fathy A S, EL-Maddah I and EL-Mahallawy (2014): Acute poisoning related deaths reported at toxicology unit of Tanta Emergency University Hospital. Master Thesis at Faculty of Medicine, Tanta University, 59-60

El-Sarnagawy G (2017): Predictive factors of mortality in acute aluminum phosphide poisoning: 5 years retrospective study in Tanta Poison Control Unit. Ain Shams Journal of Forensic Medicine and Clinical Toxicology, 29(2), 70-79.

El-Sheikh A, Hashem A, Elgohary M, Elfadl A A and Lashin H (2017): Evaluation of the potential cardiotoxic effects in acute organophosphate toxicity as a prognostic factor. Tanta Medical Journal, 45(3), 115.

Farahat T M, Shaheen H M, Sanad Z F and Frag N A (2016): Knowledge, attitudes, and practices of organophosphorus pesticide exposure among women affiliated to the Manshat Sultan Family Health Center (rural area) in Menoufia governorate: an intervention study. Menoufia medical journal, 29(1), 115. 
Farooqui W A, Uddin M, Qadeer R and Shafique K (2020): Trajectories of vital status parameters and risk of mortality among acute organophosphorus poisoning patients-a latent class growth analysis. BioMed Central public health, 20(1), 1-9.

Fliers E, Alkemade A and Wiersinga W M (2001): The hypothalamic-pituitary-thyroid axis in critical illness. Best Practice and Research Clinical Endocrinology and Metabolism, 15(4), 453-464.

Ghonem M M, Lashin H I, Hodeib A A and Soliman N A (2018): L-Carnitine as an Adjuvant Treatment in Acute Organophosphorus Pesticides Poisoning: A Randomized Clinical Trial. Mansoura Journal of Forensic Medicine and Clinical Toxicology, 26(2), 37-52.

Goad R T, Goad J T, Atieh B H and Gupta R C (2004): Carbofuran-induced endocrine disruption in adult male rats. Toxicology mechanisms and methods, 14(4), 233-239.

Gundogan K, Donmez-Altuntas H, Hamurcu Z, Akbudak I H, Sungur M, Bitgen N and Bayram F (2018): Evaluation of chromosomal DNA damage, cytotoxicity, cytostasis, oxidative DNA damage and their relationship with endocrine hormones in patients with acute organophosphate poisoning. Mutation Research / Genetic Toxicology and Environmental Mutagenesis, 825, 1-7.

Gunnell D, Eddleston M, Phillips M R and Konradsen F (2007): The global distribution of fatal pesticide self-poisoning: systematic review. BioMed Central Public Health, 7(1), 357.

Güven M, Bayram F, Ünlühizarci K and Kelestimur F (1999): Endocrine changes in patients with acute organophosphate poisoning. Human and experimental toxicology., 18(10), 598-601.

Herman JP, McKlveen J M, Ghosal S, Kopp B, Wulsin A, Makinson R, Scheimann $\mathrm{J}$ and Myers B (2016): Regulation of the HypothalamicPituitary-Adrenocortical Stress Response. Comprehensive Physiology Journal, 6 (2), 603621.

Hoffman RS, Nelson L S, Goldfrank L R, Howland MA, Lewin NA and Flomenbaum NE (2011): Initial Evaluation of the Patient: Vital Signs and Toxic Syndromes. In: Flomenbaum N E (ed): Gold frank's toxicologic emergencies (9th edition). Chapter 3. McGrawHill Professional, New York. 36-41

Huang HS, Lee KW, Ho CH, Hsu CC, Su SB, Wang JJ and Huang CC (2017): Increased risk for hypothyroidism after anticholinesterase pesticide poisoning: a nationwide population-based study. Endocrine, 57(3), 436-444.

Hulse E J, Davies J O, Simpson A J, Sciuto A M and Eddleston M (2014): Respiratory complications of organophosphorus nerve agent and insecticide poisoning. Implications for respiratory and critical care. American journal of respiratory and critical care medicine, 190(12), 1342-1354.
Hundekari I A, Surykar A N, Dongre N N and Rathi D B (2012): Acute Poisoning with Organophosphorus Pesticide: Patients Admitted to A Hospital in Bijapur, Karnataka. Journal of Krishna Institute of Medical Sciences University, 1, 38-47.

Kale B (2014): A review on organophosphorus toxicity in the farmers of solapur district from India. International Journal of Biological Research, 2(2), 69-77.

Karki P, Ansari J A, Bhandary S and Koirala S (2004): Cardiac and electrocardiographical manifestations of acute organophosphate poisoning. Singapore medical journal, 45, 385389.

Kronenberg H M (2008): Classification and pathophysiology of cushing's syndrome. In: Melmed S, Polonsky K and Larsen PR (Eds): Williams Textbook of Endocrinology $\left(11^{\text {th }}\right.$ Edition). Philadelphia: Saunders, Elsevier. Pp 445-504.

Kumar S V, Sasikala V M and Kumar G V (2010): A study on poisoning cases in a tertiary care hospital. Journal of Natural Science, Biology, and Medicine, 1(1), 35-39.

Lee S, Han J W and Kim E S (2013): Butyrylcholinesterase deficiency identified by preoperative patient interview. Korean journal of anesthesiology, 65(6 Suppl), S1

Liu H X, Liu C F and Yang W H (2015): Clinical study of continuous micropump infusion of atropine and pralidoxime chloride for treatment of severe acute organophosphorus insecticide poisoning, Journal of the Chinese Medical Association. 78(12): 709-713.

Luo K, Zhang R, Aimuzi R, Wang Y, Nian M and Zhang J (2020): Exposure to Organophosphate esters and metabolic syndrome in adults. Environment International, 143, 105941.

Martin-Reina J, Duarte J A, Cerrillos L, Bautista J D and Moreno I (2017): Insecticide reproductive toxicity profile: organophosphate, carbamate and pyrethroids. $J$ Toxins, 4(1), 1-7.

Moussa M, Mohamed S, Hilal M, Elnabi M and Zaki N (2018): Predictive Value of Triage Vital Signs and Conscious Level for Outcome Evaluation in Acutely Organophosphate Poisoned Patients. Ain Shams Journal of Forensic Medicine and Clinical Toxicology, 31(2), 33-40.

Najari F, Dadpour B, Vahabzadeh M, AlizadehGhamsari A, Mousavi S R and Kial I B (2018): value of blood lactate and tsh in predicting length of hospitalization and outcome of poisoned patients admitted to the ICU. Pharmacophore, 9(3), 7-12.

Nield L and Kamat D (2011): Fever. In: Kliegman R, Stanton B, Geme J, Schor N, Behrman R (eds.): Nelson text book of pediatric $\left(19^{\text {th }}\right.$ edition). Elsevier Saunders Inc: Philadelphia. Chapter 170. 896-899.

Ohbe H, Jo T, Matsui H, Fushimi K and Yasunaga H (2018): Cholinergic crisis caused by 
cholinesterase inhibitors: a retrospective nationwide database study. Journal of Medical Toxicology, 14(3), 237-241.

Oreby M and El-Madah E (2017): Prediction of Acute Organophosphate Poisoning Using Glasgow Coma Sale, Serum Cholinesterase and S100B. Ain Shams Journal of Forensic Medicine and Clinical Toxicology,28(1), 100107.

Persson H, Sjöberg G, Haines J and Pronczuk de Garbino J (1998): Poisoning Severity Score ( Pss ) Ipcs / Eapcct. Clinical Toxicology, 36, 205-213.

Peter J V, Sudarsan T I and Moran J L (2014): Clinical features of organophosphate poisoning: A review of different classification systems and approaches. Indian journal of critical care medicine: peer-reviewed, official publication of Indian Society of Critical Care Medicine, 18(11), 735.

Phanindra M D, Satyanarayana P V V and Acharya A (2020): A prospective evaluation of correlation between cholinesterase level and severity of organophosphorus poisoning. International Journal of Advances in Medicine, 7(2), 276.

Pisano S R, Howard J, Posthaus H, Kovacevic A and Yozova I D (2017): Hydrocortisone therapy in a cat with vasopressor-refractory septic shock and suspected critical illness-related corticosteroid insufficiency. Clinical Case Report, 5 (7), 11231129.

Rabha M, Baruah C and Boro M (2017): Clinical profile of acute organophosphorous poisoning with special reference to serum creatine phosphokinase and serum lactate dehydrogenase levels as prognostic markers: a tertiary care hospital based observational study in north-east India. International Journal of Medical Research Professionals. 3(6), 83-88.

Rao B N and Bhavana R (2015): Organophosphorous intoxication and hyperthyroidism. International Journal of Research in Medical Sciences, 3(10), 2857.

Roberts D M and Brett J (2014): Clinical Management of Acute OP Pesticide Poisoning. In: BalaliMood, M. and M. Abdollahi (eds.): Basic and Clinical Toxicology of Organophosphorus Compounds. Springer London, London. 141175.

Samprathi A, Chacko B, D'sa S R, Rebekah G, Vignesh Kumar C, Sadiq M and Peter J V (2020): Adrenaline is effective in reversing the inadequate heart rate response in atropine treated organophosphorus and carbamate poisoning. Clinical Toxicology, 1-7.

Sarhan N (2015): APACHE II, SAPS II, and SOFA Scoring Systems Used for Predicting Outcome in Acute Organophosphate Intoxicated Patients Admitted to the Intensive Care Unit-Poison
Control Center Ain Shams University Hospitals (A Prospective Study). Ain Shams Journal of Forensic Medicine and Clinical Toxicology, 25(2), 110-119.

Satar S, Sebe A, Topal M and Karcioglu O (2004): Endocrine effects of organophosphate antidotal therapy. Advances in therapy, 21(5), 301-311.

Shadnia S, Zamani N, Hassanian-Moghaddam H, Shafaroodi H, Padandar M and Rezaeizadeh M H (2018): Prognostic value of cortisol and thyroid function tests in poisoned patients admitted to toxicology ICU. World journal of emergency medicine, 9(1), 51.

Şimşek Z Ö, Sevim M, Şimşek Y, Sungur M, Gündoğan K and Güven M (2019): Effects of Organophosphate Poisoning on the Endocrine System in the Long Term: A Pilot Study. Erciyes Medical Journal/Erciyes Tip Dergisi, 41(1), 33-36.

Someswar G, Reddy Y, Kumari V S, Gupta A and Prabhakarrao R (2015): Study of Clinical Profile of Organophosphate Compound Poisoning with Special Reference to Electrocardiographic Changes and Electrolyte Derangements. Indian Journal of Mednodent and Allied Sciences, 3(1), 12-17.

Sungur M and Güven M (2001): Intensive care management of organophosphate insecticide poisoning. Critical care, 5(4), 211.

Tattersall J E (2018): Anticholinesterase toxicity. Current Opinion in Physiology, 4, 4956.

Tomescu D, Cobilinschi C, Tincu R C, Totan A, Neagu T P, Diaconu C C and Macovei R A (2018): Changes of Thyroid Hormonal Status in Organophosphate Exposure. metabolism, 12, 13.

Uddin F, Rizwan N, Waheed $\Psi$ K and Zakria K (2020): Electrocardiographic Changes in Patients of Organophosphorus Compound Poisoning. International Journal of Multidisciplinary and Current Research, 8 , 2321-3124

Vadiveloo T, Donnan P T, Murphy M J and Leese G P (2013): Age-and gender-specific TSH reference intervals in people with no obvious thyroid disease in Tayside, Scotland: the Thyroid Epidemiology, Audit, and Research Study (TEARS). The Journal of Clinical Endocrinology and Metabolism, 98(3), 11471153.

Yang F W, Zhao G P, Ren F Z, Pang G F and Li Y X (2020): Assessment of the endocrine-disrupting effects of diethyl phosphate, a nonspecific metabolite of organophosphorus pesticides, by in vivo and in silico approaches. Environment International, 135, 6-8. 


\section{تقييم دور هرموني الكورتيزول و تحفيز الغدة الدرقية كعوامل للتنبؤ في المرضي المصابين بالتسمم الحاد

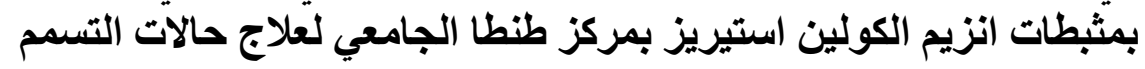

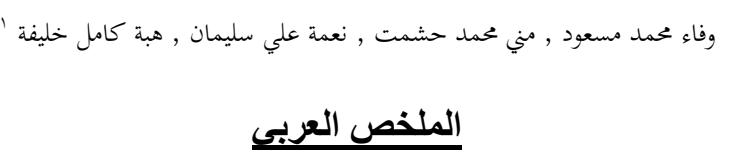

المقدمة: يعتبر التشمم بمضادات الكولينستيراز مشكلة عالمية كبرى تؤدي إلى وفاة الآلاف كل عام. التتبؤ بمصبر الحالات امر يهم

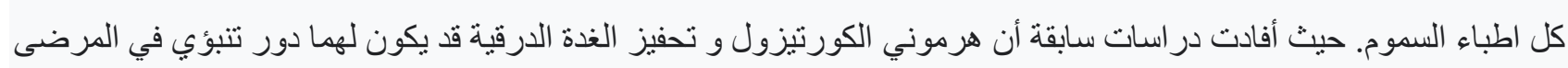
الذين أصييوا بالتسمم بالفو سفات العضوي. الهلف: هدفت هذه الدراسة إلى تقييم دور هرموني الكورتيزول و تحفيز الغدة الدرقية للتنبؤ بمصير حالات التسمم بالحاد بالمبيدات

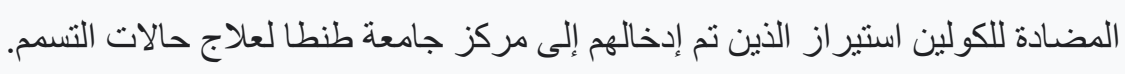
الطريقة المستخدمة في البحث: تضمنت هذه الدراسة وج مريضًا يعانون من التسمم الحاد بمضادات الكولينستيراز والذين كانوا

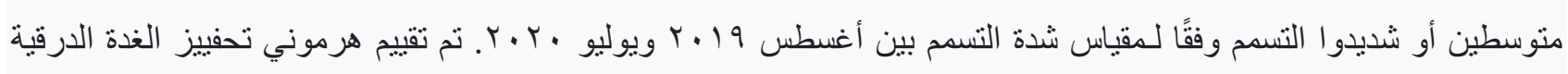
والكورتيزول التسلسلي بواسطة تقنيات ELISA.

النتائج : كانت نتائج هذه الدراسة أن هرمون تحفييز الغدة الدرقية ليس له دور تنبؤي ولكن للكورتيزول دور تنبؤي في الوفيات والحاجة إلى دخول وحدة العناية المركزة.

الاستتناج: قياس الكورتيزول عند دخول المستشفى ، بعد ست و اثنتي عثرة ساعة بمكن أن يتنبأ بالحاجة إلى دخول وحدة العناية المركزة و الوضع على جهاز التتفس الصناعي، في حين أن قياسه عند الدخول الي المستشفي يمكن أن يكون مؤشرًا للوفيات. 